\title{
Brucellosis Ontology (IDOBRU) as an extension of the Infectious Disease Ontology
}

Yu Lin ${ }^{1,2,3}$, Zuoshuang Xiang ${ }^{1,2,3}$ and Yongqun $\mathrm{He}^{1,2,3^{*}}$

\author{
* Correspondence: \\ yongqunh@umich.edu \\ ${ }^{1}$ Unit for Laboratory Animal \\ Medicine, University of Michigan \\ Medical School, Ann Arbor, MI \\ 48109, USA \\ Full list of author information is \\ available at the end of the article
}

\begin{abstract}
Background: Caused by intracellular Gram-negative bacteria Brucella spp., brucellosis is the most common bacterial zoonotic disease. Extensive studies in brucellosis have yielded a large number of publications and data covering various topics ranging from basic Brucella genetic study to vaccine clinical trials. To support data interoperability and reasoning, a community-based brucellosis-specific biomedical ontology is needed.
\end{abstract}

Results: The Brucellosis Ontology (IDOBRU: http://sourceforge.net/projects/idobru), a biomedical ontology in the brucellosis domain, is an extension ontology of the core Infectious Disease Ontology (IDO-core) and follows OBO Foundry principles. Currently IDOBRU contains 1503 ontology terms, which includes 739 Brucella-specific terms, 414 IDO-core terms, and 350 terms imported from 10 existing ontologies. IDOBRU has been used to model different aspects of brucellosis, including host infection, zoonotic disease transmission, symptoms, virulence factors and pathogenesis, diagnosis, intentional release, vaccine prevention, and treatment. Case studies are typically used in our IDOBRU modeling. For example, diurnal temperature variation in Brucella patients, a Brucella-specific PCR method, and a WHO-recommended brucellosis treatment were selected as use cases to model brucellosis symptom, diagnosis, and treatment, respectively. Developed using OWL, IDOBRU supports OWL-based ontological reasoning. For example, by performing a Description Logic (DL) query in the OWL editor Protégé 4 or a SPARQL query in an IDOBRU SPARQL server, a check of Brucella virulence factors showed that eight of them are known protective antigens based on the biological knowledge captured within the ontology.

Conclusions: IDOBRU is the first reported bacterial infectious disease ontology developed to represent different disease aspects in a formal logical format. It serves as a brucellosis knowledgebase and supports brucellosis data integration and automated reasoning.

\section{Background}

Brucellosis is a zoonotic infectious disease caused by intracellular Gram-negative bacteria Brucella spp. Since its initial isolation from tissue of a deceased patient by Dr. David Bruce in 1887 [1], Brucella has been found in many animals, including cattle, pigs, goat, sheep, dogs, fish and so on. Human brucellosis remains the most common zoonotic disease worldwide, with more than 500, 000 new cases reported annually [2]. The variety of its clinical manifestations makes diagnosis and treatment difficult. Currently there is no available human brucellosis vaccine. As a select agent

(C) 2011 Lin et al; licensee BioMed Central Ltd. This is an Open Access article distributed under the terms of the Creative Commons Attribution License (http://creativecommons.org/licenses/by/2.0), which permits unrestricted use, distribution, and reproduction in any medium, provided the original work is properly cited. 
categorized by the USA Centers for Disease Control and Prevention (CDC), aerosolized Brucella can also be used for bioterrorism [3].

Many online resources for Brucella information are available. For example, Brucella Bioinformatics Portal (BBP) is a web portal that allows users to search and analyze individual Brucella genes and link to more than 20 existing databases and analysis programs [4]. PATRIC Pathosystems resource supports browsing, visualization, and detailed analyses of Brucella genomes [5]. VIOLIN vaccine database and analysis system stores information of licensed Brucella vaccines and vaccine candidates [6]. The Brucella data collected in these resources has been widely used for various purposes by researchers around the world. However, the following bottlenecks prevent advanced data exchange and integration among these online resources: (1) frequent use of different terminologies for the same concepts, (2) a lack of logical and machine-readable relations among different terms, and (3) a lack of machine-readable and communitysupported data exchange format (e.g., the OWL format) for representation of the Brucella data. These obstacles prevent computer-assisted automated reasoning.

Biomedical ontologies are sets of hierarchical terms and relations that represent entities in the biomedical science and show how these entities relate to each other. To support automated reasoning, ontological terms are often expressed in formal logic, together with documentation and definitions [7-9]. Biomedical ontologies play important roles in areas such as knowledge management (including data indexing and information retrieval); data integration, exchange and semantic interoperability; and decision support and reasoning [10]. To facilitate translational medical research in infectious diseases, an Infectious Disease Ontology (IDO) Consortium http://www.infectiousdiseaseontology.org/ has been established. IDO is aimed to develop a suite of interoperable ontologies that jointly cover the entire infectious disease domain, spanning infectious disease specialties and the clinical care, public health, and biomedical research domains. The IDO suite of ontologies are developed using a core-extension approach in which disease- or pathogen-specific ontologies are developed as extension ontologies from a common IDO core [11]. The IDO-core ontology provides coverage of those entities relevant to infectious diseases generally, including terms such as host, pathogen, focal infection, and herd immunity. Meanwhile, the IDO extensions cover entities relevant to specific disease or pathogen types. The IDOcore was developed within the framework of the Basic Formal Ontology (BFO) [9] and the Ontology of General Medical Sciences (OGMS) [12]. The IDO-core terms have simple and formal natural language definitions as well as formal logical expressions stated in terms of relations from the OBO relation ontology and terms from IDO or other OBO ontologies, such as the Gene Ontology (GO) [7]. Specific IDO extension ontologies (e.g., Brucellosis Ontology, Influenza Ontology [13], and Malaria Ontology [14]) are maintained by experts from specific infectious disease areas. Different IDO extension ontologies are developed from the core in a coordinated fashion that ensures interoperability among all IDO extension ontologies.

In this paper, we report our development of a Brucellosis Ontology (IDOBRU) as an IDO-core extension ontology that targets the brucellosis domain.

\section{Results}

In what follows, italics are used to refer to a specific term within IDOBRU where appropriate. Prefix of an existing ontology is listed before one term, in such a form as 
OBI:data item. By default, the IDOBRU terms in this paper have no prefix. Additional file 1 provides detailed information of all ontology terms and relations used in this manuscript.

\section{IDOBRU general design and introduction}

IDOBRU is an ontology that covers and crosses the biomedical domains of clinical care, public health and biomedical research in the specific brucellosis field. IDOBRU contains the vocabulary and terms from seven major aspects: host infection and zoonotic disease transmission, symptoms, virulence factors and pathogenesis, diagnosis, intentional release, vaccine prevention, and treatment. The goal of IDOBRU is to establish a knowledgebase of Brucella and brucellosis. The targeted users of IDOBRU include brucellosis researchers, microbiologists, bioinformaticians, clinicians, governments and related decision makers.

The current IDOBRU version "Arbor Release" (version number 1.1.41 released on October 28, 2011) contains a total of 1503 terms, including 1469 classes, 26 object properties, and 8 datatype properties. Among them, 414 terms are IDO-core-specific terms. In total, IDOBRU includes 739 IDOBRU-specific terms, including 726 classes, 5 object properties and 8 datatype properties. Following the principles of OBO Foundry, IDOBRU has reused or adopted external ontologies that are OBO Foundry ontologies and candidate ontologies. IDOBRU fully imports the whole BFO, RO and IDO-core. Using the OntoFox [15] software program, IDOBRU imports external terms from eight other existing ontologies and resources: Chemical Entities of Biological Interest (CHEBI) [16], Gene Ontology [7], Information Artifact Ontology (IAO) [17], NCBI Taxonomy database [18], Ontology for Biomedical Investigations (OBI) [19], Ontology for General Medical Science (OGMS) [12], Protein Ontology [20], and Vaccine Ontology (VO) [21] (Table 1). In total, IDOBRU has imported 764 terms from 11 external ontologies. Figure 1 shows the major architecture of IDOBRU that includes key top-level terms in IDOBRU. As shown in this figure, all brucellosis-specific terms are subclasses of terms from higher level ontologies including the IDO-core, OGMS, OBI, VO, and GO.

According to an agreement between IDOBRU and the IDO-core development teams, a "hub-and-spoke" model is adopted for the development of the IDO-core and IDO extension ontologies. Specifically, the IDO-core acts as the hub and plays a role as the mediator for all extension ontologies, and infectious disease extension ontologies act as spokes. As a principal in OBO foundry ontologies, an identifier is always bipartite, in the form of ID-space:Local-ID, for example: IDO:0000001. Instead of using different ID-spaces and Local-IDs for individual IDO extension ontologies, every extension ontology uses the same ID-space: "IDO", as the IDO-core. For example, an ID used for an IDOBRU term is IDO:0100246, instead of IDOBRU:0000246. Unique ID blocks pre-assigned by the IDOcore team are used to differentiate specific extension ontologies. This strategy has many advantages: (1) to avoid duplicate terms and efforts; (2) to enforce the same best practice for ontology development across different extension ontologies; and (3) to encourage better collaboration and closer coordination between the IDO-core and IDO extension development teams. Meanwhile, each extension ontology is developed independently and maintains its own development repository site.

In the following sections, we will introduce IDOBRU representation of different aspects of brucellosis in details. 
Table 1 IDOBRU specific terms and terms imported to IDOBRU from 11 source ontologies

\begin{tabular}{|c|c|c|c|c|c|}
\hline$\#$ & Ontology & Classes & $\begin{array}{l}\text { Object } \\
\text { Properties }\end{array}$ & $\begin{array}{l}\text { Datatype } \\
\text { Properties }\end{array}$ & Total \\
\hline 1 & IDOBRU (Brucellosis Ontology) specific & 726 & 5 & 8 & 739 \\
\hline \multicolumn{6}{|c|}{ Imported ontologies as whole } \\
\hline 1 & BFO (Basic Formal Ontology) & 39 & 0 & 0 & 39 \\
\hline 2 & RO (Relation Ontology) & 0 & 9 & 0 & 9 \\
\hline 3 & IDO (Infectious Disease Ontology) & 411 & 3 & 0 & 414 \\
\hline 4 & $\begin{array}{l}\text { OGMS (Ontology for General Medical } \\
\text { Science) }\end{array}$ & 82 & 0 & 0 & 82 \\
\hline \multicolumn{6}{|c|}{ Imported from other external ontologies } \\
\hline 1 & OBI (Ontology for Biomedical Investigations) & 12 & 7 & 0 & 19 \\
\hline 2 & NCBITaxon (NCBI Taxonomy) & 103 & 0 & 0 & 103 \\
\hline 3 & IAO (Information Artifact Ontology) & 7 & 2 & 0 & 9 \\
\hline 4 & VO (Vaccine Ontology) specific & 68 & 0 & 0 & 68 \\
\hline 5 & $\begin{array}{l}\text { CHEBI (Chemical Entities of Biological } \\
\text { Interest) }\end{array}$ & 9 & 0 & 0 & 11 \\
\hline 6 & GO (Gene Ontology) & 9 & 0 & 0 & 9 \\
\hline 7 & PRO (Protein Ontology) & 1 & 0 & 0 & 1 \\
\hline Total & 12 & 1469 & 26 & 8 & 1503 \\
\hline
\end{tabular}

\section{Modeling brucellosis, host infections and pathogen transmission in IDOBRU}

The genus Brucella is taxonomically placed in the alpha-2 subdivision of the class NCBITaxon:Proteobacteria. Traditionally there are eight species of Brucella based on the preferential host specificity: B. melitensis (goats), B. abortus (cattle), B. suis (swine),

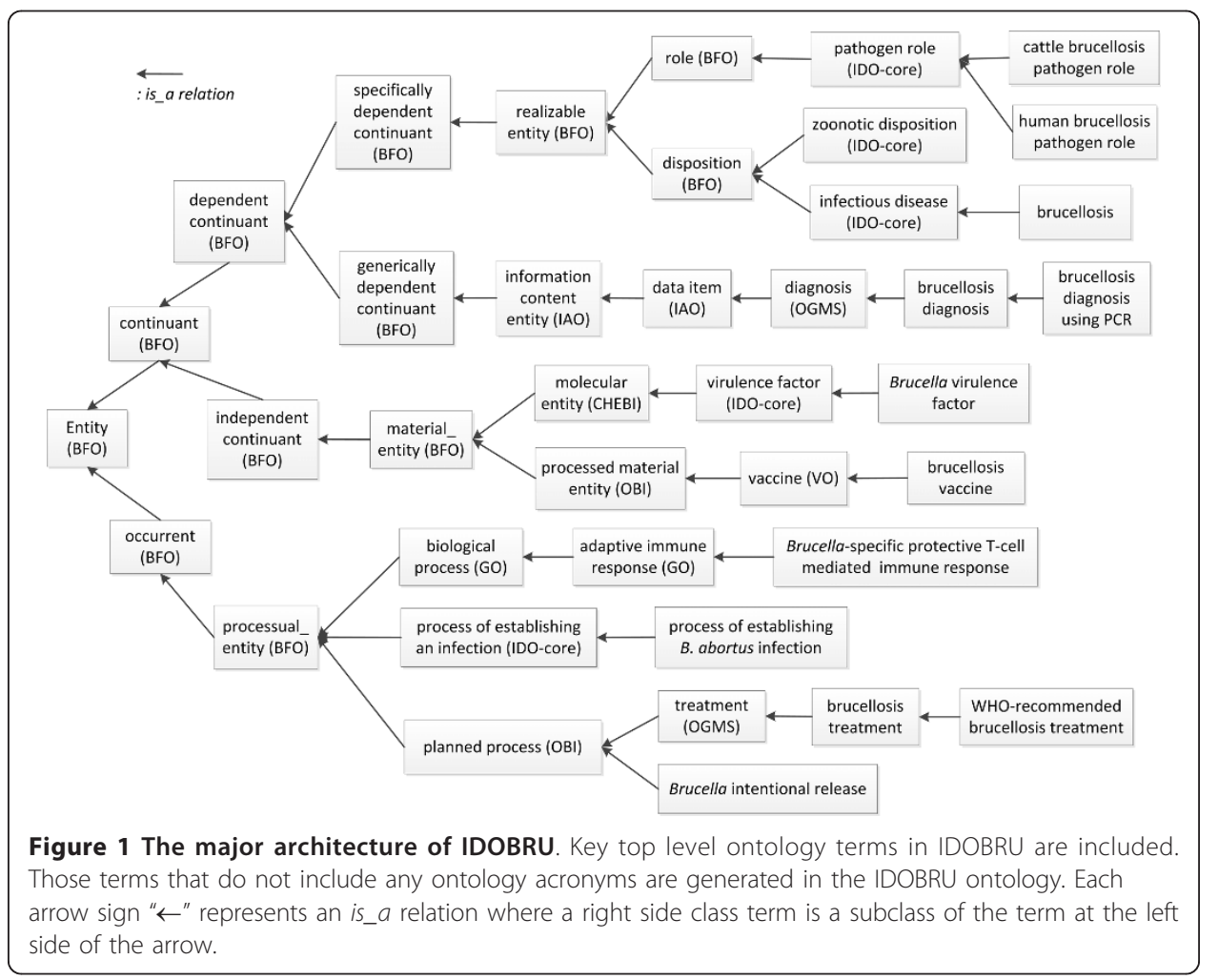


B. canis (dogs), B. ovis (sheep), B. neotomae (desert mice) [4], B. cetaceae (cetacean), and $B$. pinnipediae (seal) [22]. The first four species are pathogenic to humans in decreasing order of severity, making brucellosis a zoonotic disease.

The zoonosis feature of brucellosis is captured by the term of IDO:zoonotic disposition. In IDO, zoonotic disposition is defined as an infectious disposition that is the disposition to be transmitted from an infected, non-human host to a human host [23]. Therefore, in IDOBRU, Brucella is asserted as bearer of IDO:zoonotic disposition (Figure 2). For example, B. abortus, a subclass of Brucella, can infect both cattle and human. As subclasses of brucellosis pathogen role, both of the human brucellosis pathogen role and cattle brucellosis pathogen role are roles of B. abortus. During the process of establishing B. abortus infection, the zoonotic disposition of Brucella is realized. An infected human or cattle is the host of Brucella during the infection. Therefore, both Brucella infected human and Brucella infected cattle are the bearer of Brucella host role. Brucella infected human (or cattle) has disposition of human (or cattle) brucellosis, which is realized in the disease course of human (or cattle) brucellosis (Figure 2).

To model the complicated infection and animal-to-human transmission mechanisms in brucellosis, we used the following scenario as an example. A brucellosis human patient is infected via drinking the unpasteurized Brucella-contaminated milk produced by a Brucella-infected cow. The Brucella-infected cow produces milk that is contaminated with Brucella during the Brucella-contaminated milk producing process, where the Brucellacontaminated milk producing disposition is realized. The Brucella-contaminated milk, a specified output of this producing process, is drunk by the Brucella infected human when he/she realizes a drinking function (Figure 2).

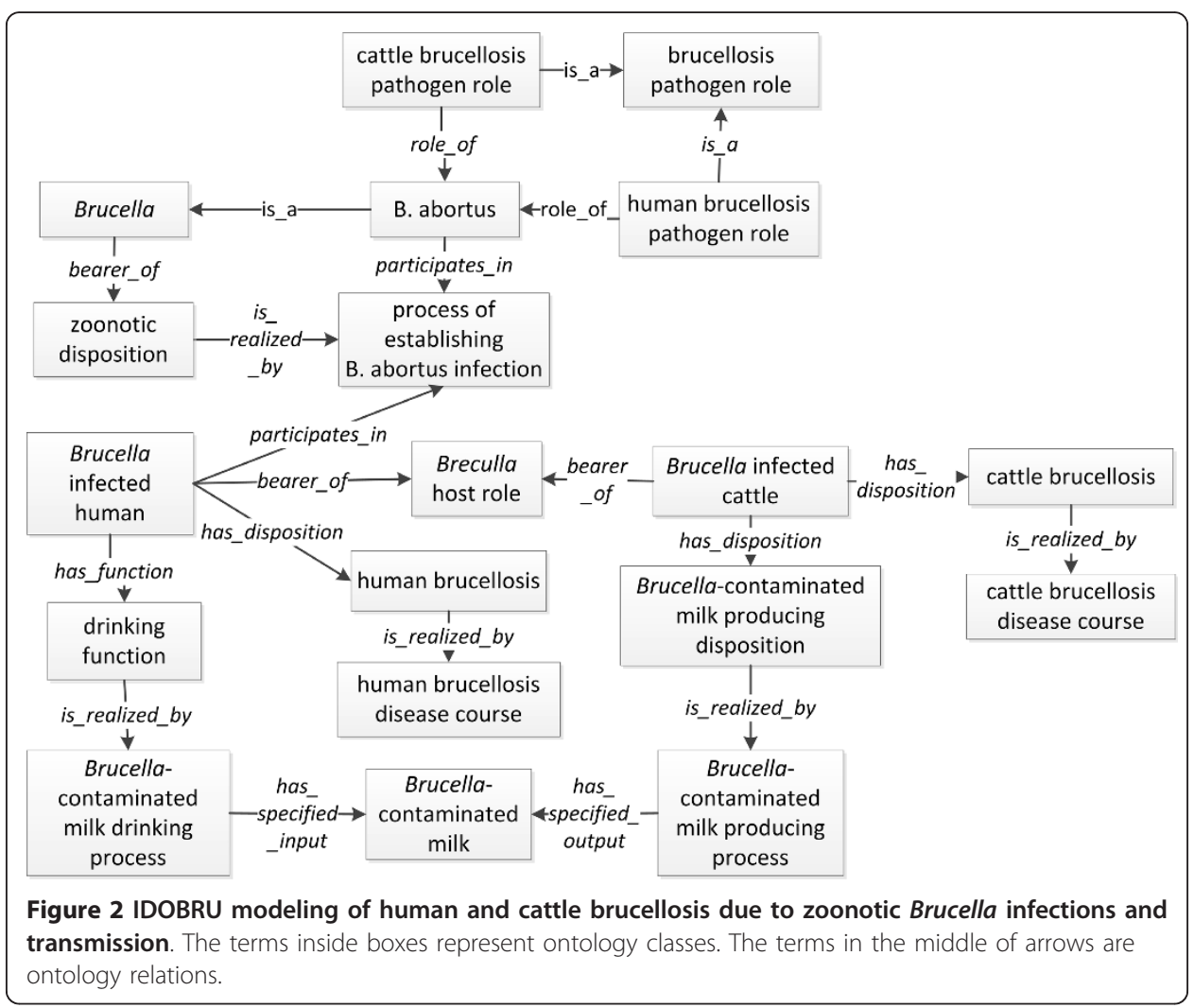




\section{Modeling Brucellosis symptoms in IDOBRU}

A symptom is a bodily feature that a patient observes. The brucellosis symptom is a subclass of OGMS:symptom [12]. Brucellosis usually causes abortion and sterility in non-human animals. Undulant fever, myalgia, and arthralgia of the large joints are the main symptoms in human brucellosis patients [24]. As a use case, the human undulant fever is modeled here (Figure 3). Undulant fever is a term with an ambiguous meaning. It has been used as an alternative synonym for human brucellosis, as well as a symptom of human brucellosis. In IDOBRU's point of view, the reality of the undulant fever is based on the fact that during the course of the disease, brucellosis patient's temperature shows diurnal variation [25]. As a scenario of the temperature diurnal variation process, a brucellosis human patient has a quality of elevated temperature measured as $40^{\circ} \mathrm{C}$ in the afternoon, and then has a quality of normal temperature measured as $37.1^{\circ} \mathrm{C}$ in the morning. Both temperature elevation in the afternoon and temperature staying normal in the morning are parts of the process of diurnal variation of temperature, which is a part of the human brucellosis disease course. The human brucellosis symptom is a quality of brucellosis human patient. At the different time points, the human patient has different temperature qualities as measured with different temperature numbers. It is noted that specific temperatures in different time points fall into instance levels of our ontology rather than class levels of entity. However, the elevated temperature and normal temperature without temporal part are considered as subclasses of BFO:Quality at the class level.

\section{Modeling Brucella virulence factors and pathogenesis in IDOBRU}

Brucella doesn't bear the classic bacterial virulence factors as capsules, secreted proteases, exotoxins, endotoxins, etc. As an intracellular bacterium, Brucella virulence relies on its ability to survive and replicate in the vacuolar compartments of macrophages. The interaction between Brucella and macrophages is critical for the

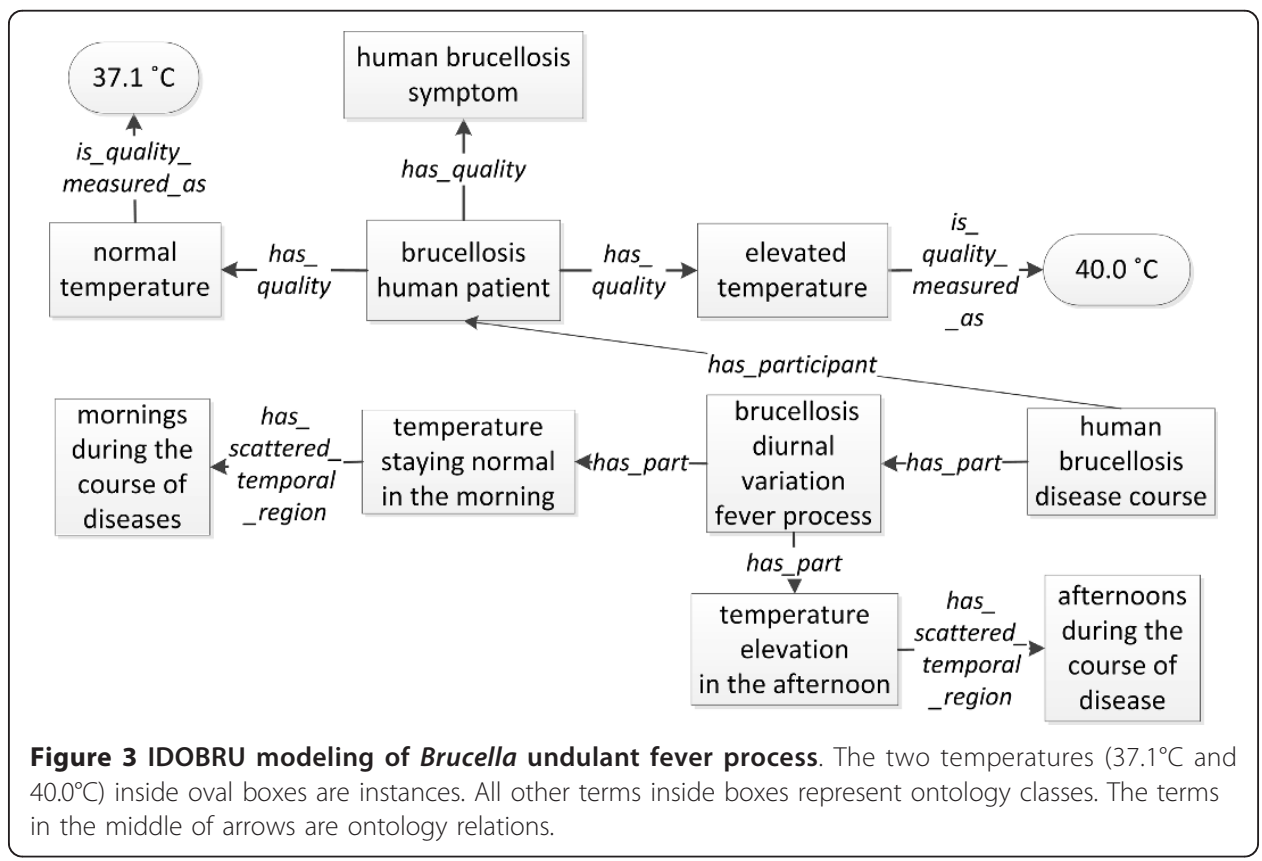


establishment of a chronic infection [26,27]. Three processes are critically important in order for Brucella to establish a successful infection: (a) the entry into host cell, (b) the survival and (c) the replication within membrane-bound compartments in host cells, particularly macrophages. The type IV secretion system encoded by Brucella virB operon is required for the survival of Brucella inside replicative phagosomes in macrophages [28]. Brucella lipopolysaccharide (LPS) of smooth virulent Brucella strains is required to prevent the phagosome-lysosome fusion in macrophages, allowing the survival and replication of Brucella inside macrophages [28,29]. Brucella VirB1 protein and Brucella LPS, the two important virulence factors, are used as examples for virulence factor modeling in IDOBRU (Figure 4).

In IDO-core, the terms related to virulence factor are IDO: virulence factor and IDO: virulence factor disposition. IDO:virulence factor is the bearer of IDO: virulence factor disposition. As an IDO extension, IDOBRU has Brucella virulence factor and Brucella virulence factor disposition. Using the Brucella-macrophage interaction as a scenario, the Brucella virulence factor disposition is realized in the process of establishing Brucella infection in macrophages. As a process entity, the process of establishing Brucella infection in macrophages has three partial processes: establishment of Brucella intracellular localization, Brucella survival in macrophages, and Brucella replication in macrophages (Figure 4). The 'part of' relation (RO:part_of) has been asserted between each of these partial processes and the process of establishing Brucella infection in macrophage. Brucella VirB1, a subtype of Brucella protein, has the disposition as a Brucella virulence factor disposition and participates in the processes of Brucella survival in macrophages and Brucella replication in macrophages. Brucella LPS participates in the processes of Brucella replication in macrophages, and Brucella entry into macrophages, which is a partial process of establishment of Brucella intracellular localization.

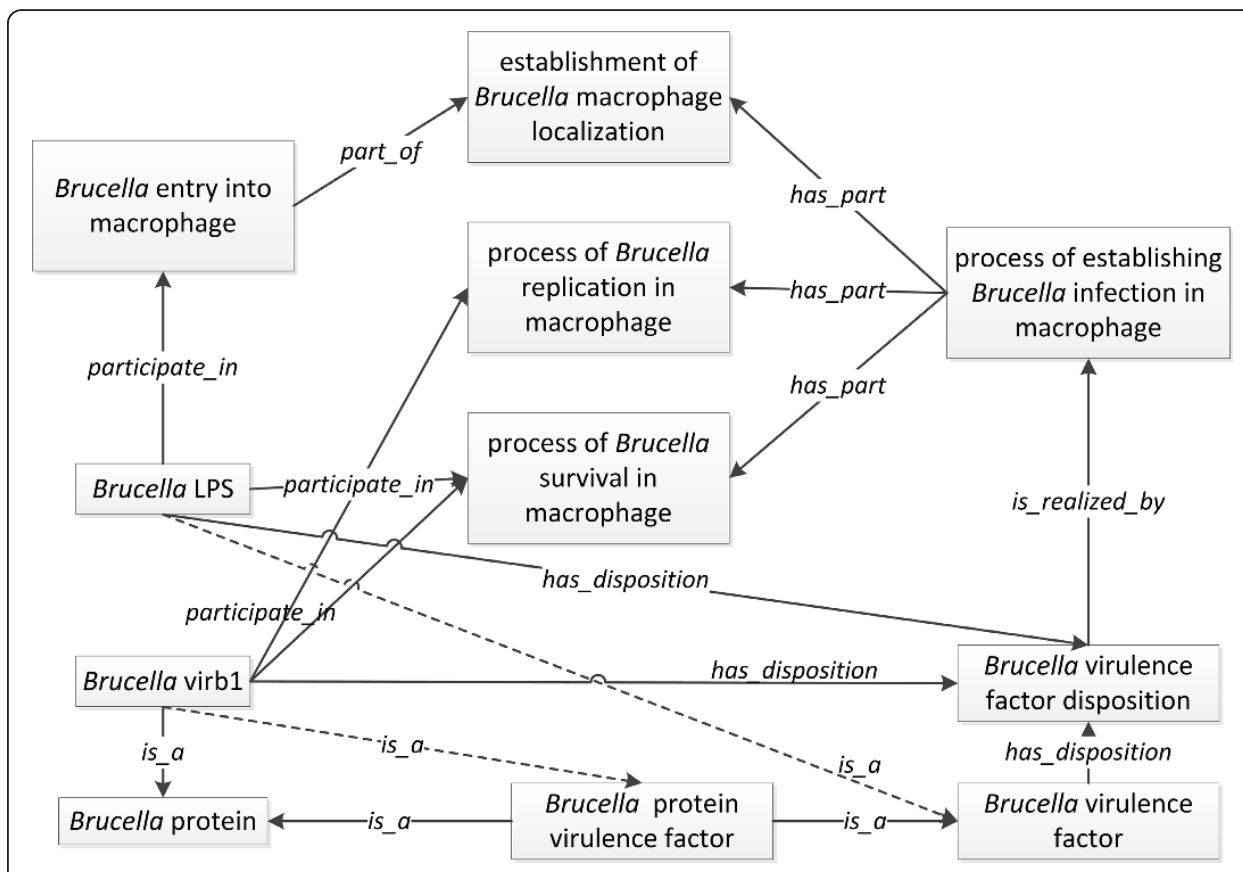

Figure 4 IDOBRU modeling of Brucella virulence factors and pathogenesis. The terms inside boxes represent ontology classes. The terms in the middle of arrows are ontology relations. 
In IDOBRU, the virulence factors can be different types of molecular material such as protein and lipopolysaccharide. We asserted a term Brucella protein virulence factor as a subtype of Brucella virulence factor in IDOBRU. The formal logic definitions of Brucella virulence factor and Brucella protein virulence factor have been given as such:

Brucella virulence factor $\equiv$ (part_of some Brucella) and (bearer_of some 'Brucella virulence factor disposition')

Brucella protein virulence factor $\equiv$ 'Brucella protein' and (bearer_of some 'Brucella virulence factor disposition')

After reasoning, Brucella VirB1 has been computed as a subclass of Brucella protein virulence factor, and Brucella LPS as a subclass of Brucella virulence factor.

In total, 245 curated Brucella virulence factors have been imported into IDOBRU from a MySQL database of the Brucella Bioinformatics Portal (BBP) [4].

\section{Modeling Diagnosis of brucellosis in IDOBRU}

Diagnosis of human brucellosis cannot be made solely on clinical grounds due to the wide variety of its clinical manifestations. It is essential to confirm the diagnosis using bacteriological and serological tests. Many assays (e.g., polymerase chain reaction or PCR, ELISA, and agglutination assays) have been used for diagnosis of brucellosis [25]. The use of PCR allows for rapid and accurate diagnosis, and it is a rapid method to confirm the infection of Brucella [30]. IDOBRU adopts the terminological framework of OGMS for a top level representation of diseases, such as disease causes and manifestations, diagnosis, and other disease interpretations used in the clinic. According to OGMS, diagnosis is defined as "a conclusion of an interpretive process that has as input a clinical picture of a given patient and as output an assertion" [12]. Brucellosis diagnosis is a subclass of OGMS:diagnosis. Based on different assays used in the diagnostic process, brucellosis diagnosis has many subclasses, e.g., brucellosis diagnosis by PCR test, brucellosis diagnosis by ELISA, and brucellosis diagnosis by microscopy.

PCR assays have been frequently used for diagnosis. A PCR assay is different from PCR amplification. The PCR amplification is a material transformation process with an output of amplified PCR product. A PCR assay is designed for specific purposes (e.g. detecting a target sequence for diagnosis) using PCR amplification. The PCR amplification is a part of a PCR assay. To model brucellosis diagnosis, a PCR assay used to test a Brucella gene omp-2 encoding for an outer membrane protein (OMP-2) from patient's blood sample is specifically studied [31] (Figure 5). First, the IDOBRU term PCR assay for detecting Brucella omp-2 was asserted as a subclass of OGMS:laboratory test. Using brucellosis patient derived specimen as specified input, the PCR assay will generate an output that is the laboratory finding of Brucella omp-2 detection. As a subclass of IAO: information content entity, this specific laboratory finding is about the patient derived specimen. The IAO relation is_about relates an information artifact to an entity. Secondly, this specific PCR assay has an integral part: PCR to amplify 193 bp region in Brucella OMP-2 gene [31]. OBI:polymerase chain reaction has been imported from OBI, and the PCR amplification of 193 bp region in Brucella OMP-2 gene is a subclass of OBI:polymerase chain reaction in IDOBRU. The inputs of the PCR amplification are: (a) Brucella OMP-2 forward primer, (b) Brucella OMP-2 reverse primer, and (c) DNA extracted from Brucella contaminated blood specimen. The output of this PCR amplification is the product of PCR amplification of $169 \mathrm{bp}$ region in Brucella OMP-2. Finally, only a positive 


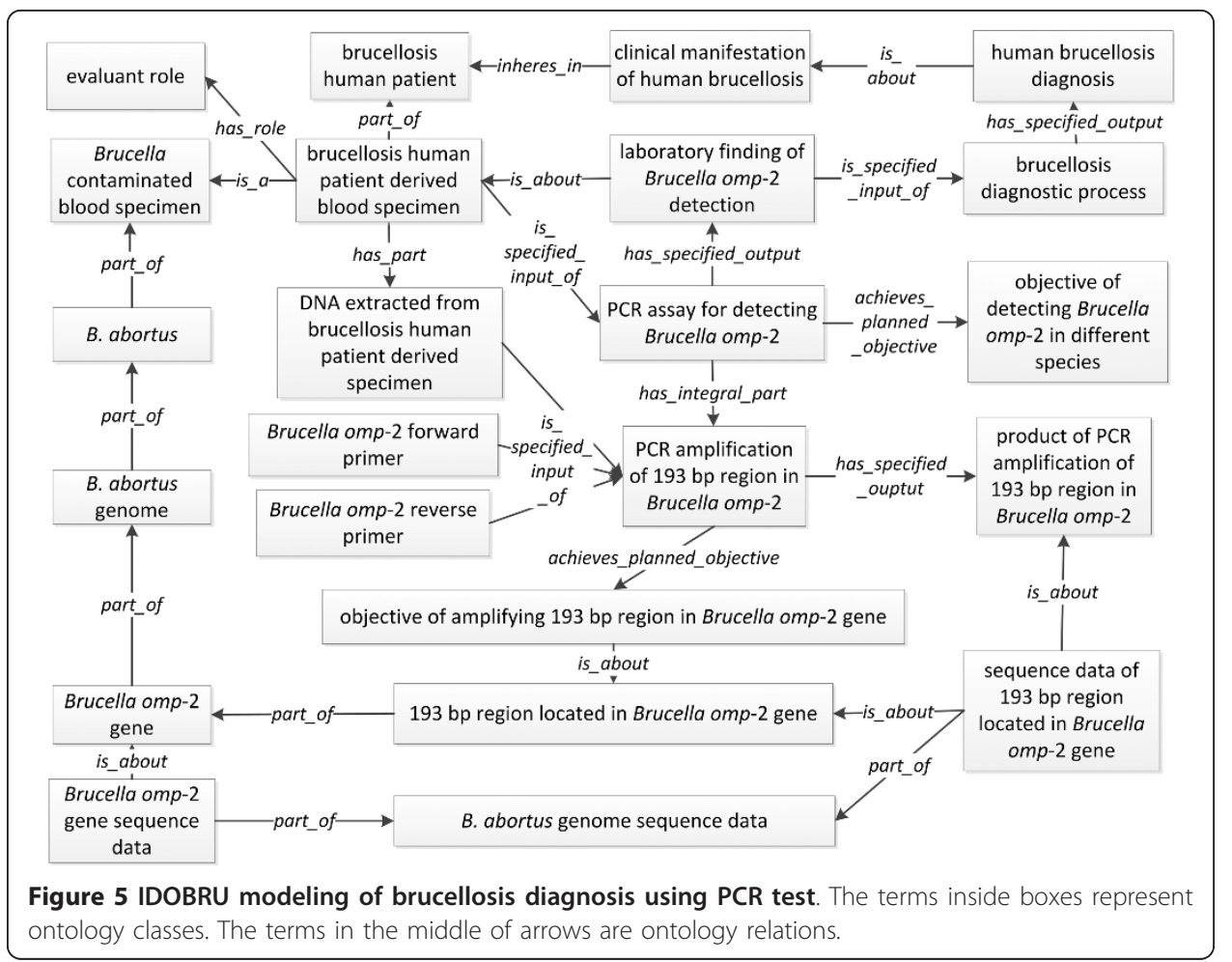

result obtained from the PCR assay would allow a doctor to draw a diagnostic conclusion of Brucella infection. Using the laboratory finding of Brucella OMP-2 detection as an input for the brucellosis diagnostic process, the output is human brucellosis diagnosis. This diagnosis thus is_about the clinical manifestation of brucellosis (Figure 5).

The primer sequence information and PCR product described in above PCR experiment are modeled as primer sequence data and PCR product sequence data, respectively. All of the sequence data of primers and PCR product are parts of B. abortus genome sequence data [31]. The relation denotes is defined in IAO that allows us to assert the relationship between digital data of a DNA sequence and its corresponding physical biological material sequence. In our case, PCR sequence data (a digital data item) denotes PCR product of the Brucella OMP-2 PCR amplification. A 'part-whole' relation is used in two different types of entities here: a) The material entity: OMP-2 gene as part of $B$. abortus genome, which is part of the B. abortus bacterial cell that exists in the blood specimen taken from the human patient. b) The information content entity: the OMP-2 gene sequence data as part of $B$. abortus genome sequence data.

\section{Modeling brucellosis epidemiology and intentional release in IDOBRU}

Many factors can affect the prevalence of brucellosis in various species of livestock. Prevalence of brucellosis can vary according to climatic conditions, geography, species, sex, age and diagnostic tests applied [32]. Although it has been eradicated in many developed countries in Europe, Australia, Canada, Israel, Japan and New Zealand, brucellosis remains an uncontrolled problem in regions of high endemicity such as the Africa, Mediterranean, Middle East, parts of Asia and Latin America. Re-emergence of brucellosis is reported in Japan 1996, Bulgaria during 2005 to 2007 and FBH (Federation of Bosnia and Herzgovina) recently [25]. 
In IDOBRU, terms such as eradication of brucellosis, Brucella accidental release, brucellosis endemic site, brucellosis free site, brucellosis non-endemic site, brucellosis surveillance, and more others, have been used to capture the knowledge of epidemiology aspect of brucellosis. Since brucellosis is a zoonotic disease, the above classes have subclasses both in human brucellosis and non-human brucellosis.

Brucella can be intentionally used as a bioterrorism weapon as an incapacitating agent rather than a lethal agent. Although brucellosis causes low fatality rate, human brucellosis is a notoriously debilitating disease, and brucellosis patients require prolonged treatment [24]. Brucella organisms can be aerosolized and released in infectious doses, such as a sum of 10-500 virulent aerosol Brucella organisms. It is important to disinfect Brucella once its release is identified. Most commercial disinfectants are effective at killing or neutralizing Brucella organisms. Bleach (10\%) has been used as an effective disinfectant to control the release of Brucella.

The intentional release of aerosolized Brucella is modeled (Figure 6). Both Brucella and aerosolized Brucella has the role as a bioterrorism agent. The process of Brucella intentional release is a OBI:planned process. The aerosolized Brucella has been produced by the Brucella aerosolization process (a subtype of OBI:planned process), which is an integral part of the Brucella intentional release process. When Brucella is used for the intentional release, Brucella is a specified input in the process of Brucella intentional release. When a Brucella bioterrorism attack occurs, the bleach disinfection of aerosolized Brucella process can be initiated for the purpose of disinfection. The bleach that bears a disinfectant role is a specified input in this process (Figure 6).

\section{Modeling vaccine prevention of brucellosis in IDOBRU}

Vaccination is the most effective means of reducing brucellosis in cattle, sheep and goat [25]. Currently IDOBRU imports 66 Brucella vaccines from the Vaccine Ontology (VO; http://www.violinet.org/vaccineontology[21], including four licensed animal Brucella vaccines and 62 Brucella vaccine candidates that have been proven effective in laboratory animal models [33]. Currently, there is no licensed human brucellosis vaccine. Available animal vaccines may cause disease and are considered unsuitable for use in humans. Nonetheless, a human Brucella vaccine is needed to protect the public against human brucellosis and bioterrorism [34].

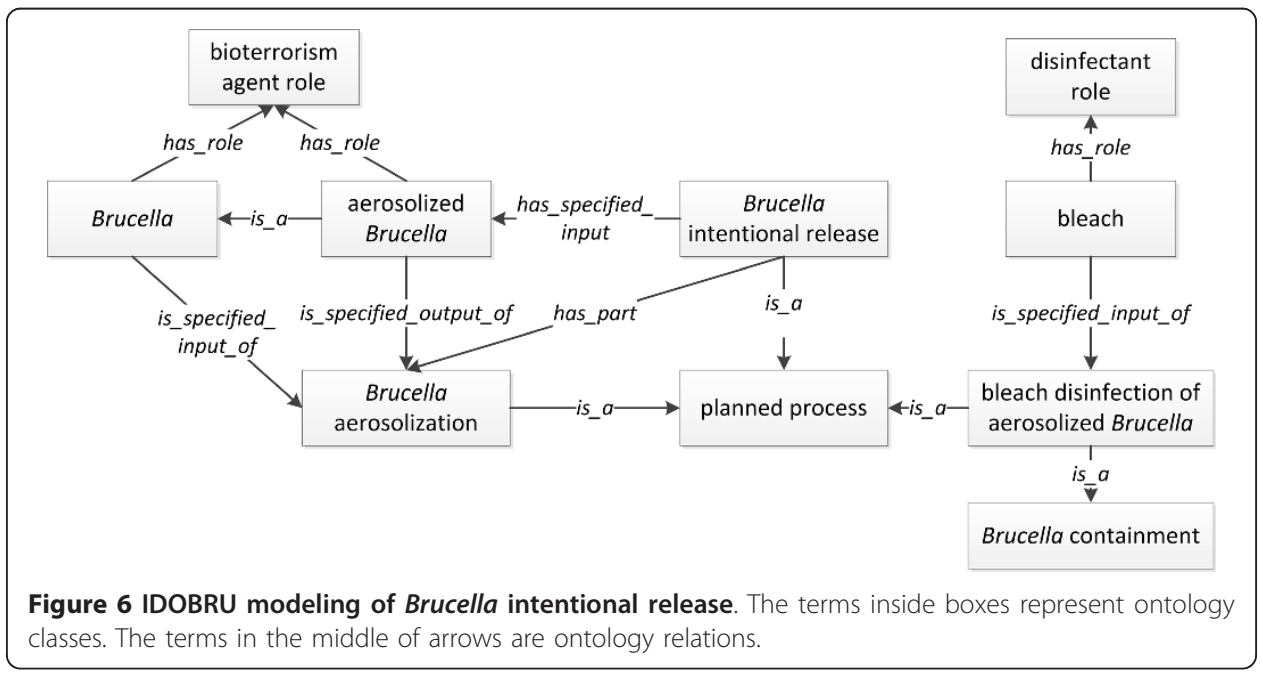


To support rational Brucella vaccine design, it is important to understand the protective immunity induced by protective Brucella antigens. A protective antigen can stimulate protective adaptive immune response against Brucella infection, and is used as an active component of a vaccine. In IDOBRU, this Brucella-specific adaptive immune response is a subclass of GO: adaptive immune response, which is a GO:immune response (Figure 7). For example, Brucella $\mathrm{Cu} / \mathrm{Zn}$ superoxide dismutase (SodC), coded by Brucella sodC gene, is a known Brucella protective antigen (bearer of the Brucella protective antigen role) [35-37]. Brucella sodC gene has been used for as part of the $B$. abortus DNA vaccine pcDNA-SOD [38] (Figure 7). Currently IDOBRU has imported 21 Brucella protective antigens from VO.

\section{Modeling treatment of brucellosis in IDOBRU}

Once brucellosis is diagnosed, a treatment will follow. The World Health Organization (WHO) has recommended a standard method for treatment of uncomplicated brucellosis cases in adults and children eight years of age and older: doxycycline $100 \mathrm{mg}$

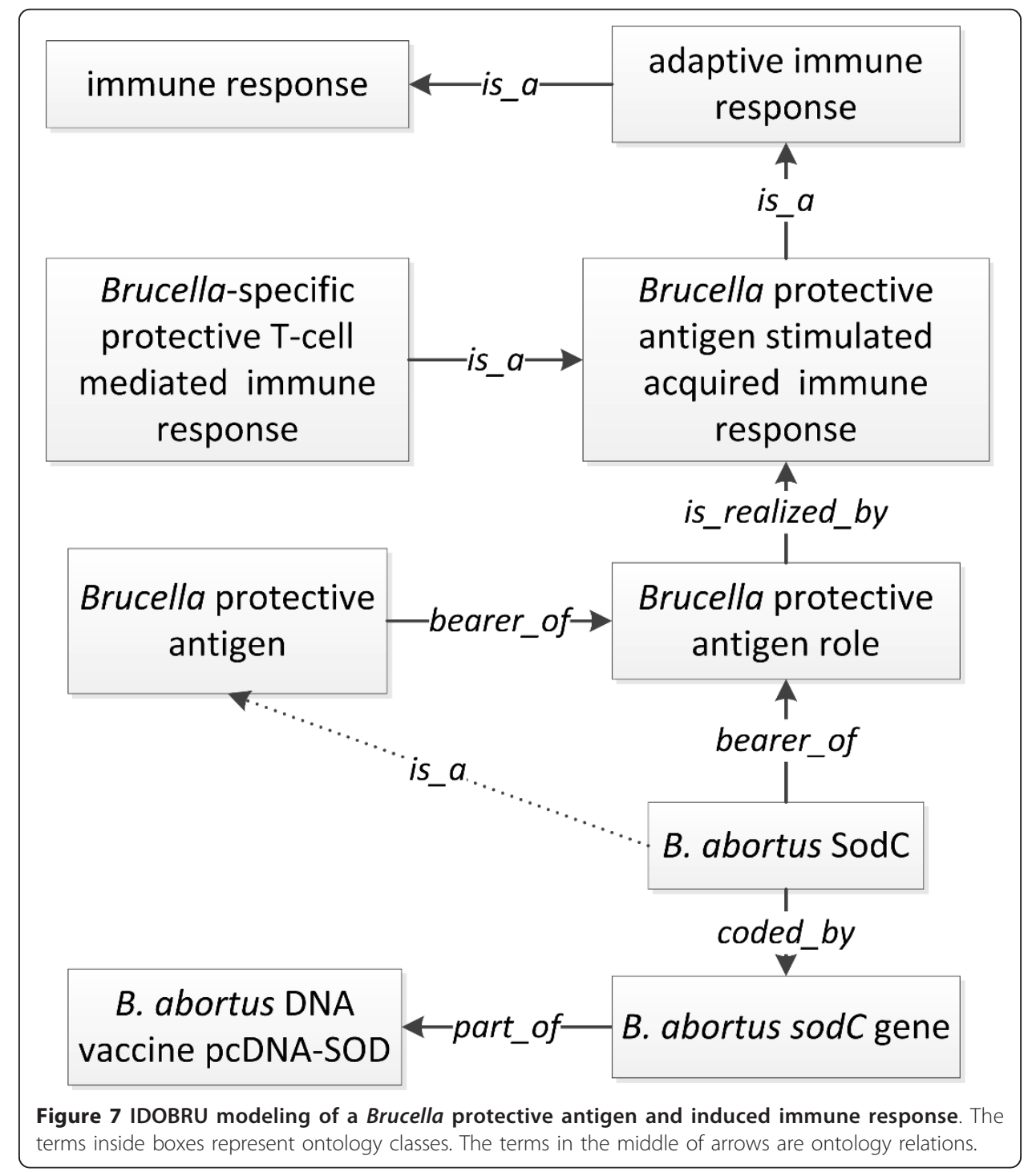


administrated twice a day for six weeks + streptomycin $1 \mathrm{~g}$ daily for two to three weeks [39]. This WHO treatment has been modeled in IDOBRU and described below (Figure 8).

The planned process WHO standard treatment for human brucellosis in adults and children eight years of age and older consists of two integral parts: 1) WHO standard doxycycline treatment for human brucellosis; 2) WHO standard streptomycin treatment for human brucellosis. Using doxycycline treatment as an example, administrating doxycycline $100 \mathrm{mg}$ twice a day is part of the example process. Its specified input is the material entity $100 \mathrm{mg}$ doxycycline with a quality of dose measured as $100 \mathrm{mg}$. A datatype property, hasDailyfrequency, has been used for representing the frequency of the administration of doxycycline and its value is 2 . The required duration of the whole treatment, is represented by the term continuous 6 weeks treatment duration, which is a subclass of BFO:connected temporal region. Here we adopted the definition of connected temporal region asserted in BFO version 1.1: "A temporal region [span:TemporalRegion] every point of which is mediately or immediately connected with every other point" [40]. Similar to the administration frequency, the 6 weeks' duration is modeled by another datatype property: 'hasDurationinDays value " 42 "^^ integer' [41]. Since the treatment is limited to patients whose age is greater than 8 years' old, in IDOBRU, the age of a human patient is captured by the datatype property "hasAge" with a "float type" restriction. Then, the patient who is suitable for this treatment will be represented by the following constrain as: 'human patient' and (hasAge some float [> = "8"^^float]).

\section{Ontology reasoning using IDOBRU}

Since IDOBRU is developed using the OWL format, OWL-based automated reasoning can be done using reasoning programs within OWL editors or by developing new SPARQL query and reasoning programs. As an example, we designed the following

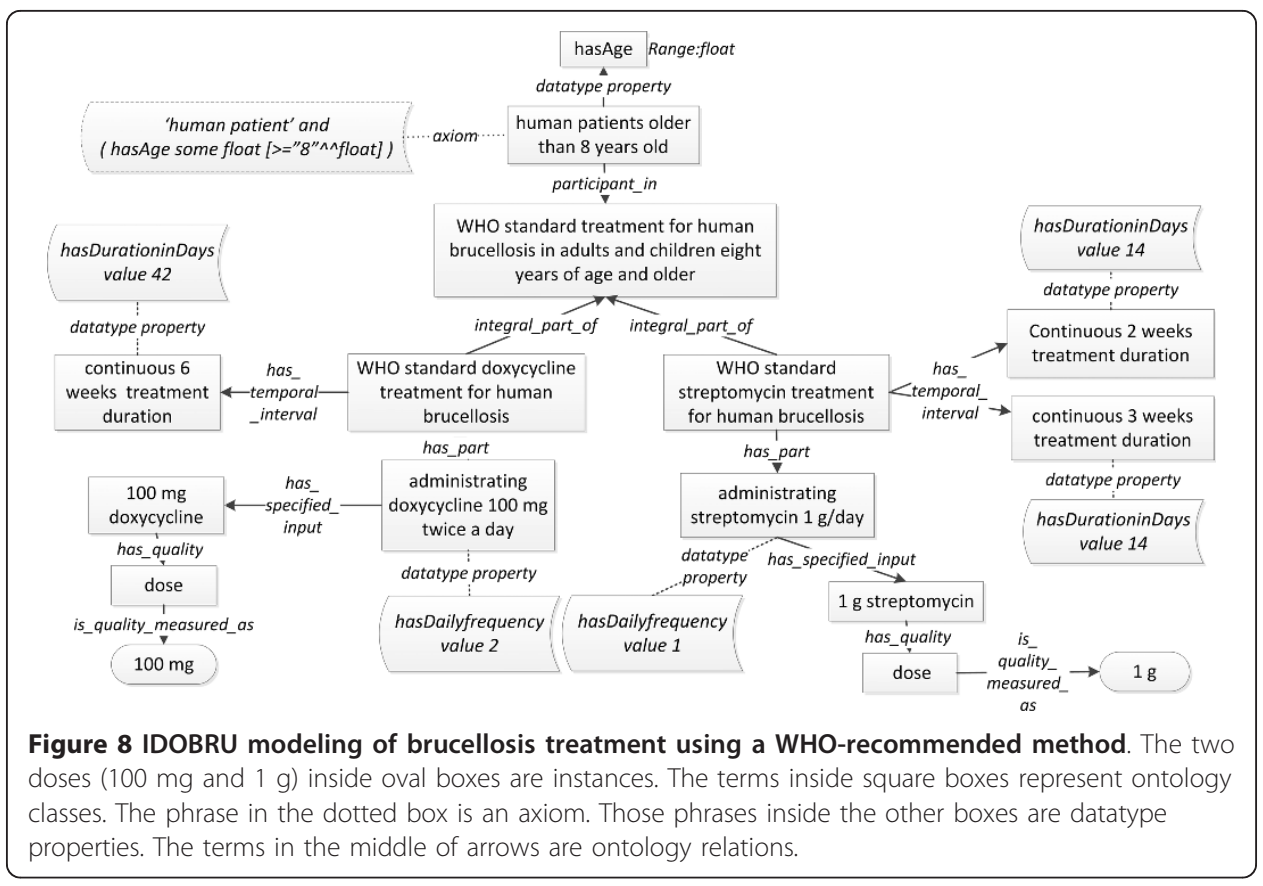


question for querying the ontology: "What Brucella virulence factors are also protective antigens?" In IDOBRU, a Brucella virulence factor is logically defined as "something that is a bearer of some Brucella virulence factor disposition". A protective antigen is logically defined as "something that is a bearer of some Brucella protective antigen role". To answer this question, the following query was performed in the DL Query tab of the OWL editor Protégé 4:

(bearer_of some 'Brucella protective antigen role') and (bearer_of some 'Brucella virulence factor disposition')

The answers to this query are: Brucella lipopolysaccharide and Brucella sodC and their six subclasses: Brucella abortus lipopolysaccharide, Brucella abortus SodC, Brucella melitensis lipopolysaccharide, Brucella melitensis SodC, Brucella ovis lipopolysaccharide and Brucella suis lipopolysaccharide (Figure 9).

We have also developed an IDOBRU SPARQL query interface at http://www.phidias. us/bbp/idobru/sparql/index.php. A SPARQL query script has been generated to obtain the same answers by querying the same question (see Additional file 2).

\section{Discussion}

Our approach of developing IDOBRU combines a top-down and bottom-up realism methodology [42]. In terms of the top-down method, we started by making IDO-core

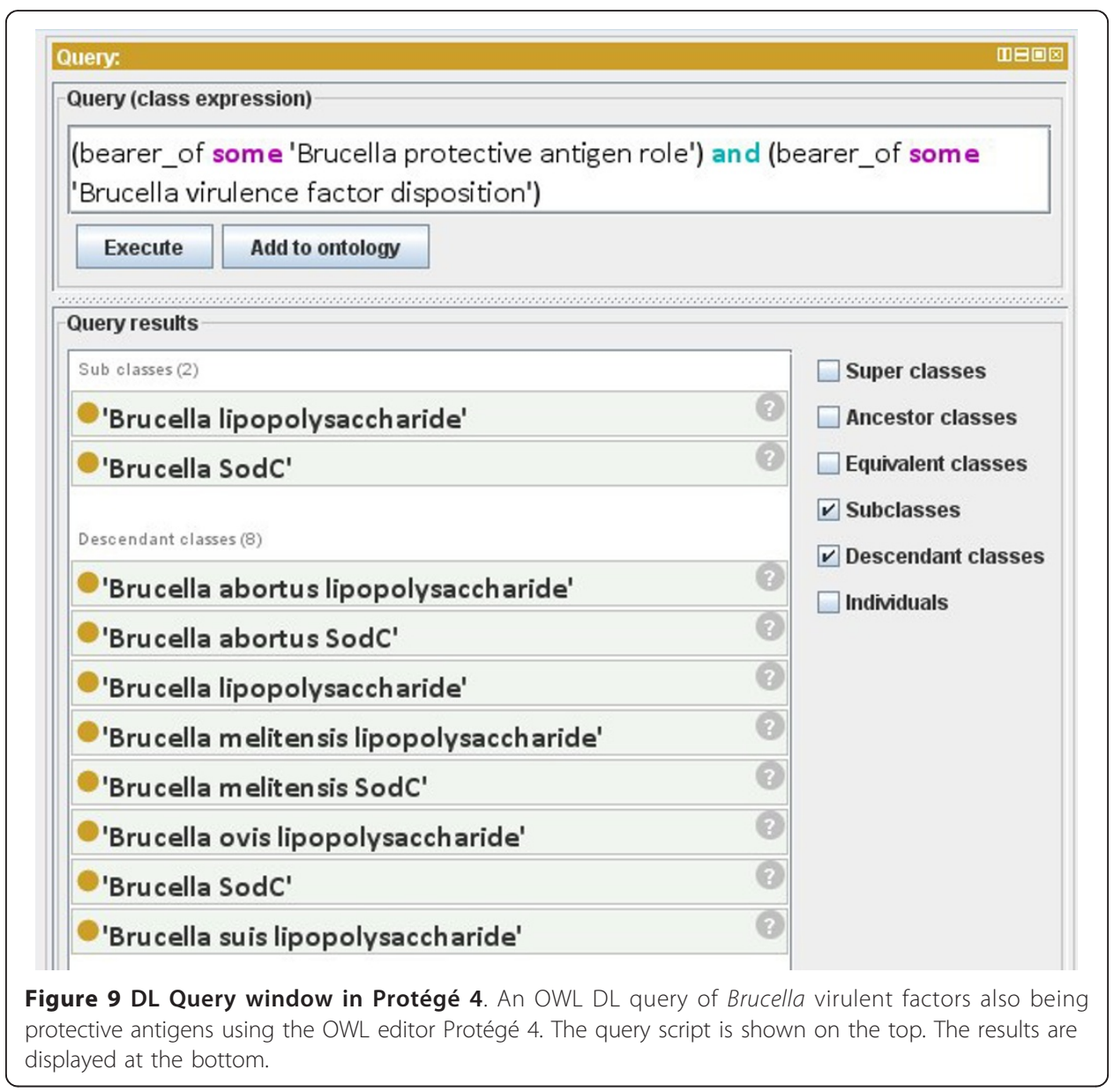


as our direct upper ontology, and most of the IDOBRU terms have their respective IDO-core terms as super-classes. The bottom-up method is to identify the most specific terms by using scenarios, and then generalize them into higher ontology classes. The prior knowledge of upper ontologies and existing ontologies, especially $\mathrm{BFO}, \mathrm{RO}$, IDO, OGMS, VO, and OBI, is essential for the development of IDOBRU. Before a new term is generated, we shall check whether or not this new term and its possible upper level term have existed in other ontologies. Extensive discussion is often needed to achieve a consensus in new term definition.

The limitations of upper ontologies limit ontology development in IDOBRU. For example, the ontological theory of signs and symptoms and their relations to pathological processes has not been well-developed yet. OGMS is in the very early stages of addressing this need [12]. Since it is one of the most important features of the clinical manifestation of human brucellosis, IDOBRU tried to model the undulant fever process (Figure 3). After several rounds of debating and discussing in the community of IDO, finally the agreement has been achieved: (a) the term elevated temperature is a BFO:Quality, and it is the quality of the human brucellosis patient; and (b) the brucellosis diurnal variation fever process is a subclass of OGMS: pathological bodily process. IDOBRU will evolve while other ontologies make their progress.

IDOBRU also has its own limitations. First, IDOBRU is still at the early stage of its development. As a knowledgebase, the information about brucellosis in IDOBRU is still limited. For example, while we have modeled one PCR assay for brucellosis diagnosis in IDOBRU, many other brucellosis diagnostic assays have been reported in the literature and collected in online resources including the BBP [4]. We also need to model other diagnosis assays including many serological diagnosis methods. Another limitation of IDOBRU exists in its coverage of epidemiological terms. During the course of our IDOBRU development, a large number of epidemiological terms have been generated. Among them, many terms (e.g., infectious disease endemic site) are not available in current IDO-core. Other IDO extension ontologies (e.g., Influenza Ontology) have also been developing their epidemiological terms. Therefore, closer collaborations among the IDO-core and IDO extension ontologies are needed.

Currently, several IDO extensions are being developed, such as Malaria Ontology [14], Influenza Ontology [13], Staphylococcus aureus Ontology, and Tuberculosis Ontology http://infectiousdiseaseontology.org/page/Extensions. Malaria Ontology was reported and published before an IDO-core was released [14]. Staphylococcus aureus Ontology was one of the first bacterial extension ontologies developed and has been well integrated with the IDO-core. Both S. aureus and M. tuberculosis are Gram-positive bacteria. So far Brucella has been the only Gram-negative bacterium being studied for the purpose of an IDO extension ontology development. IDOBRU is the first IDO extension ontology that is published after the official release of the IDO-core. It provides a useful real world example of using the IDO-core terms for development of an IDO extension. To our knowledge, IDOBRU is the first ontology that logically models several key topics of an infectious disease, including host infection, zoonotic disease transmission, symptoms, virulence factors and pathogenesis, diagnosis, intentional release relevant to bioterrorism, and treatment.

IDOBRU can be used for several applications. First of all, serving as a knowledgebase of Brucella and brucellosis, IDOBRU captures the knowledge extracted from 
biomedical bench research, clinical practices, and public health. Secondly, owing to the parseable and machine understandable nature of the ontology, IDOBRU supports Brucella and brucellosis data exchange, data integration, and automated reasoning. IDOBRU uses BFO, RO and other existing ontologies such as OBI and OGMS. Therefore, the IDOBRU information can easily be integrated with other ontologies and processed with software programs developed based on OBO Foundry Principles. Finally, the development of IDOBRU may serve as an example to model infectious diseases caused by other microbial pathogens. Once many other infectious diseases are modeled using the same framework, it is possible not only to compare different infectious diseases automatically, but also to discover new knowledge in infectious disease domain.

\section{Conclusions}

IDOBRU is a brucellosis-centric ontology and a knowledge-centric platform. IDOBRU can be used as a brucellosis knowledgebase and is applicable for brucellosis data exchange, data integration, and automated reasoning.

\section{Methods}

IDOBRU editing

IDOBRU was developed using the format of W3C standard Web Ontology Language (OWL2) http://www.w3.org/TR/owl-guide/. For efficient editing of IDOBRU, the Protégé 4.1 OWL ontology editor http://protege.stanford.edu/ was used.

\section{Existing ontology term import}

The whole ontologies of BFO, RO and IDO-core have been imported into IDOBRU using the OWL ontology importing feature. A web server OntoFox http://ontofox. hegroup.org/[15] was used to import external terms from existing ontologies to IDOBRU.

\section{IDOBRU access and licensing}

The latest version of IDOBRU is always available at http://svn.code.sf.net/p/idobru/ code/trunk/trunk/src/ontology/brucellosis.owl. In addition, IDOBRU has been deposited in the repositories of NCBO BioPortal http://purl.bioontology.org/ontology/IDOBRU. The IDOBRU source code is freely available under the Apache License 2.0. This licensing allows IDOBRU users to freely distribute and use IDOBRU.

\section{IDOBRU visualization and term search}

To make it convenient for users to browse and search the definitions and usages of IDOBRU terms and their relations, we have developed a user-friendly IDOBRU Browser using the Ontobee linked ontology browser system http://www.ontobee.org/browser/index.php?o=IDOBRU.

\section{SPARQL query of IDOBRU}

The IDOBRU SPARQL query web page is built as a software program compatible with the IDOBRU website http://www.phidias.us/bbp/idobru located in the Brucella Bioinformatics Portal (BBP; http://www.phidias.us/bbp) [4,43]. To develop this program, the IDOBRU was stored in a Virtuoso RDF store located in one HP ProLiant DL380 G6 server that runs the Red Hat Linux operating system (Red Hat Enterprise Linux 5 
server). PHP scripting language was used for developing the SPARQL web interface. The SPARQL queries provided by a user are implemented through HTTP against the RDF stores.

\section{Additional material}

Additional file 1: Ontology classes used in the manuscript. The file includes the detailed information about all ontology classes and relations used in the manuscript.

Additional file 2: SPARQL script. This SPARQL script is used to query the IDOBRU: "What Brucella virulence factors are also protective antigens?".

\section{List of abbreviations}

BFO: Basic Formal Ontology; BBP: Brucella Bioinformatics Portal; CHEBI: Chemical Entities of Biological Interest; DL; Description Logic; ELISA: Enzyme-Linked Immunosorbent Assay; GO: Gene Ontology; IAO: Information Artifact Ontology; IDO: Infectious Disease Ontology; IDO-core: Infectious Disease Ontology core; LPS: lipopolysaccharide; NCBI: The National Center for Biotechnology Information; NCBO: The National Center for Biomedical Ontology; OBI: Ontology for Biomedical Investigations; OBO: The Open Biomedical Ontologies; OGMS: Ontology for General Medical Science; OWL: Web Ontology Language; PATRIC: Pathosystems Resource Integration Center; PCR: Polymerase Chain Reaction; PHP: Hypertext Preprocessor; PO: Protein Ontology; RDF: Resource Description Framework; RO: OBO Relation Ontology; ro_proposed: RO proposed version; SPARQL: SPARQL Protocol and RDF Query Language; URI: Uniform Resource Identifier; VIOLIN: Vaccine Investigation and Online Information Network; VO: Vaccine Ontology; WHO: World Health Organization; XML: Extensible Markup Language.

\section{Acknowledgements}

This work has been supported by grant R01Al081062 from the National Institute of Allergy and Infectious Diseases. We acknowledge Dr. Salwa Ali's contribution in the development of IDOBRU by editing many epidemiologyassociated terms. We appreciate the discussions and advices provided by Alan Ruttenberg, Barry Smith, Lindsay Cowell, Richard Scheuermann, and other IDO consortium members during and after three related IDO workshops. We also appreciate active discussions and support by all OBI developers. We gratefully acknowledge the critical review and editing of this manuscript by Dr. George W. Jourdian from the University of Michigan Medical School and Dr. Lindsay Cowell from the University of Texas Southwestern Medical Center at Dallas.

\section{Author details}

${ }^{1}$ Unit for Laboratory Animal Medicine, University of Michigan Medical School, Ann Arbor, MI 48109, USA. ${ }^{2}$ Department of Microbiology and Immunology, University of Michigan Medical School, Ann Arbor, MI 48109, USA. ${ }^{3}$ Center for Computational Medicine and Bioinformatics, University of Michigan Medical School, Ann Arbor, MI 48109, USA.

\section{Authors' contributions}

YL, Primary IDOBRU developer, use case testing, drafting of manuscript. ZX, IDOBRU developer, Webmaster, software programmer, database administrator, and manuscript editing. YH, IDOBRU developer, project design and management, brucellosis domain expert, use case testing, and drafting of manuscript. All authors read and approved the final manuscript.

\section{Competing interests}

The authors declare that they have no competing interests.

Received: 26 May 2011 Accepted: 31 October 2011 Published: 31 October 2011

\section{References}

1. Madkour MM: Brucellosis. Kent: Butterworths; 1989.

2. Pappas G, Papadimitriou P, Akritidis N, Christou L, Tsianos EV: The new global map of human brucellosis. Lancet Infect Dis 2006, 6(2):91-99.

3. Davis RG: The AbCs of bioterrorism for veterinarians, focusing on Category B and C agents. J Am Vet Med Assoc 2004, 224(7):1096-1104

4. Xiang Z, Zheng W, He Y: BBP: Brucella genome annotation with literature mining and curation. BMC Bioinformatics 2006, 7:347.

5. Snyder EE, Kampanya N, Lu J, Nordberg EK, Karur HR, Shukla M, Soneja J, Tian Y, Xue T, Yoo H, Zhang F, Dharmanolla C, Dongre NV, Gillespie JJ, Hamelius J, Hance M, Huntington Kl, Jukneliene D, Koziski J, Mackasmiel L, Mane SP, Nguyen V, Purkayastha A, Shallom J, Yu G, Guo Y, Gabbard J, Hix D, Azad AF, Baker SC, et al: PATRIC: the VBI PathoSystems Resource Integration Center. Nucleic Acids Res 2007, , 35 Database: D401-406.

6. Xiang Z, Todd T, Ku KP, Kovacic BL, Larson CB, Chen F, Hodges AP, Tian Y, Olenzek EA, Zhao B, Colby LA, Rush HG, Gilsdorf JR, Jourdian GW, He Y: VIOLIN: vaccine investigation and online information network. Nucleic Acids Res 2008, , 36 Database: D923-928.

7. Ashburner M, Ball CA, Blake JA, Botstein D, Butler H, Cherry JM, Davis AP, Dolinski K, Dwight SS, Eppig JT, Harris MA, Hill DP, Issel-Tarver L, Kasarskis A, Lewis S, Matese JC, Richardson JE, Ringwald M, Rubin GM, Sherlock G: Gene ontology: tool for the unification of biology. The Gene Ontology Consortium. Nat Genet 2000, 25(1):25-29. 
8. Smith B, Ceusters W, Klagges B, Kohler J, Kumar A, Lomax J, Mungall C, Neuhaus F, Rector AL, Rosse C: Relations in biomedical ontologies. Genome Biol 2005, 6(5):R46.

9. Smith B, Ashburner M, Rosse C, Bard J, Bug W, Ceusters W, Goldberg L, Eilbeck K, Ireland A, Mungall CJ, OBI Consortium, Leontis N, Rocca-Serra P, Ruttenberg A, Sansone SA, Scheuermann RH, Shah N, Whetzel PL, Lewis S: The OBO Foundry: coordinated evolution of ontologies to support biomedical data integration. Nat Biotechnol 2007, 25(11):1251-1255.

10. Bodenreider O: Biomedical ontologies in action: role in knowledge management, data integration and decision support. Yearb Med Inform 2008, 67-79.

11. Cowell LG, Smith B: Infectious Disease Ontology. In Infectious Disease Informatics. Edited by: Sintchenko V. New York Dordrecht Heidelberg London: Springer; 2010:373-395.

12. Scheuermann R, Ceusters W, Smith B: Toward an Ontological Treatment of Disease and Diagnosis. The 2009 AMIA Summit on Translational Bioinformatics: 2009; San Francisco 116-120.

13. Influenza Ontology. [http://influenzaontologywiki.igs.umaryland.edu/wiki/index.php/Main_Page].

14. Topalis P, Mitraka E, Bujila I, Deligianni E, Dialynas E, Siden-Kiamos I, Troye-Blomberg M, Louis C: IDOMAL: an ontology for malaria. Malar J 2010, 9:230.

15. Xiang Z, Courtot M, Brinkman RR, Ruttenberg A, He Y: OntoFox: web-based support for ontology reuse. BMC Res Notes 2010, 3:175.

16. Degtyarenko K, de Matos P, Ennis M, Hastings J, Zbinden M, McNaught A, Alcantara R, Darsow M, Guedj M, Ashburner M: ChEBI: a database and ontology for chemical entities of biological interest. Nucleic Acids Res 2008, , 36 Database: D344-350.

17. IAO ontology. [http://code.google.com/p/information-artifact-ontology/].

18. Sayers EW, Barrett T, Benson DA, Bryant SH, Canese K, Chetvernin V, Church DM, DiCuccio M, Edgar R, Federhen S, Feolo M, Geer LY, Helmberg W, Kapustin Y, Landsman D, Lipman DJ, Madden TL, Maglott DR, Miller V, Mizrachi I, Ostell J, Pruitt KD, Schuler GD, Sequeira E, Sherry ST, Shumway M, Sirotkin K, Souvorov A, Starchenko G, Tatusova TA, et al: Database resources of the National Center for Biotechnology Information. Nucleic Acids Res 2009, 37 Database: D5-15.

19. Brinkman RR, Courtot M, Derom D, Fostel JM, He Y, Lord P, Malone J, Parkinson H, Peters B, Rocca-Serra P, Ruttenberg A, Sansone SA, Soldatova LN, Stoeckert CJ Jr, Turner JA, Zheng J, OBI consortium: Modeling biomedical experimental processes with OBI. J Biomed Semantics 2010, 1(Suppl 1):S7.

20. Natale DA, Arighi CN, Barker WC, Blake J, Chang TC, Hu Z, Liu H, Smith B, Wu CH: Framework for a protein ontology. BMC Bioinformatics 2007, 8(Suppl 9):S1.

21. He Y, Cowell LG, Diehl AD, Mobley H, Peters B, Ruttenberg A, Scheuermann R, Brinkman RR, Courtot M, Mungall C, Xiang Z, Chen F, Todd T, Colby LA, Rush H, Whetzel T, Musen MA, Athey BD, Omenn GS, Smith B: VO: Vaccine Ontology. The 1st International Conference on Biomedical Ontology (ICBO 2009): 2009; Buffalo, NY, USA Nature Precedings; 2009.

22. Cloeckaert A, Verger JM, Grayon M, Paquet JY, Garin-Bastuji B, Foster G, Godfroid J: Classification of Brucella spp. isolated from marine mammals by DNA polymorphism at the omp2 locus. Microbes Infect 2001, 3(9):729-738.

23. IDO ontology. [http://purl.obolibrary.org/obo/ido.owl].

24. Franco MP, Mulder M, Gilman RH, Smits HL: Human brucellosis. Lancet Infect Dis 2007, 7(12):775-786.

25. Madkour MM: Madkour's brucellosis. Berlin; London: Springer; 2001.

26. Chen F, Ding X, Ding Y, Xiang Z, Li X, Ghosh D, Schurig GG, Sriranganathan N, Boyle SM, He Y: Proinflammatory Caspase-2-Mediated Macrophage Cell Death Induced by a Rough Attenuated Brucella suis Strain. Infect Immun 2011, 79(6):2460-2469.

27. Chen F, He Y: Caspase-2 mediated apoptotic and necrotic murine macrophage cell death induced by rough Brucella abortus. PLoS One 2009, 4(8):e6830

28. Roop RM, Bellaire BH, Valderas MW, Cardelli JA: Adaptation of the Brucellae to their intracellular niche. Mol Microbiol 2004, 52(3):621-630.

29. He Y, Reichow S, Ramamoorthy S, Ding X, Lathigra R, Craig JC, Sobral BW, Schurig GG, Sriranganathan N, Boyle SM: Brucella melitensis triggers time-dependent modulation of apoptosis and down-regulation of mitochondrionassociated gene expression in mouse macrophages. Infect Immun 2006, 74(9):5035-5046.

30. Romero C, Pardo M, Grillo MJ, Diaz R, Blasco JM, Lopez-Goni I: Evaluation of PCR and indirect enzyme-linked immunosorbent assay on milk samples for diagnosis of brucellosis in dairy cattle. J Clin Microbiol 1995, 33(12):3198-3200.

31. Leal-Klevezas DS, Martinez-Vazquez IO, Lopez-Merino A, Martinez-Soriano JP: Single-step PCR for detection of Brucella spp. from blood and milk of infected animals. J Clin Microbiol 1995, 33(12):3087-3090.

32. Gul S, Khan A: Epidemiology and epizootology of brucellosis: A review. Pakistan Veterinary Journal 2007, 27(3):7..

33. He Y, Xiang Z: Bioinformatics analysis of Brucella vaccines and vaccine targets using VIOLIN. Immunome Res 2010, 6(Suppl 1):S5.

34. Perkins SD, Smither SJ, Atkins HS: Towards a Brucella vaccine for humans. FEMS Microbiol Rev 2010.

35. Yang B, Sayers S, Xiang Z, He Y: Protegen: a web-based protective antigen database and analysis system. Nucleic Acids Res 2011, , 39 Database: D1073-1078.

36. He Y, Vemulapalli R, Schurig GG: Recombinant Ochrobactrum anthropi expressing Brucella abortus $\mathrm{Cu}$, Zn superoxide dismutase protects mice against $B$. abortus infection only after switching of immune responses to Th1 type. Infect Immun 2002, 70(5):2535-2543.

37. Vemulapalli R, He Y, Cravero S, Sriranganathan N, Boyle SM, Schurig GG: Overexpression of protective antigen as a novel approach to enhance vaccine efficacy of Brucella abortus strain RB51. Infect Immun 2000, 68(6):3286-3289.

38. Munoz-Montesino C, Andrews E, Rivers R, Gonzalez-Smith A, Moraga-Cid G, Folch H, Cespedes S, Onate AA: Intraspleen delivery of a DNA vaccine coding for superoxide dismutase (SOD) of Brucella abortus induces SOD-specific CD4+ and CD8+ T cells. Infect Immun 2004, 72(4):2081-2087.

39. Corbel MJ: Brucellosis in humans and animals. World Health Organization 2006 2005, 102.

40. Grenon P: Spatio-temporality in Basic Formal Ontology. In IFOMIS reports. Edited by: Grenon P. Leipzig: Institute for Formal Ontology and Medical Information Science at the Faculty of Medicine of the University of Leipzig; 2003:89. 
41. The Manchester OWL Syntax. [http://www.co-ode.org/resources/reference/manchester_syntax/].

42. Smith B, Ceuster W: Ontological realism: A methodology for coordinated evolution of scientific ontologies. Applied Ontology 2010, 5(3-4):139-188

43. Xiang Z, Tian Y, He Y: PHIDIAS: a pathogen-host interaction data integration and analysis system. Genome Biol 2007, 8(7):R150.

doi:10.1186/2041-1480-2-9

Cite this article as: Lin et al.: Brucellosis Ontology (IDOBRU) as an extension of the Infectious Disease Ontology. Journal of Biomedical Semantics 2011 2:9.

Submit your next manuscript to BioMed Central and take full advantage of:

- Convenient online submission

- Thorough peer review

- No space constraints or color figure charges

- Immediate publication on acceptance

- Inclusion in PubMed, CAS, Scopus and Google Scholar

- Research which is freely available for redistribution 